\title{
Efecto patológico por hidrargiria en la fisiología sistémica de murinos.
}

\author{
Ayacucho 2020
}

Pathological effect of hydrargyria on murine systemic physiology. Ayacucho 2020

Efeito patológico da hidrargiria na fisiologia sistêmica murina. Ayacucho 2020

\section{ARTÍCULO GENERAL}

\author{
Jesús Javier Ñaccha Urbano \\ https://orcid.org/0000-0002-5035-4849 \\ jesus.naccha@unsch.edu.pe
}

Universidad Nacional San Cristóbal de Huamanga, Ayacucho - Perú

Recibido 10 de Enero 2022 | Arbitrado y aceptado 17 de Junio 2022 | Publicado en 11 Octubre 2022

\begin{abstract}
RESUMEN
El presente trabajo de investigación tiene como evaluar el efecto fisopatológico de la hidrargiria en estructuras sistémicas de murinos, lo cual se realizó en el laboratorio de Fisiología y Ciencias Naturales de la Facultad de Ciencias Biológicas.

Se utilizaron 12 ratones albinos Mus musculus, de la variedad Balb/c, donde se realizaron tratamientos en tres grupos experimentales y uno control. Cabe mencionar que se utilizaron 12 ratones machos $\mathrm{y}$ hembras, y la edad promedio fue de 50 días. Cada grupo experimental estuvo constituido por 3 ratones, a los cuales se les aplicó $1 \mathrm{ml}$ de bicloruro de mercurio $1 \mathrm{mg}$ para el I Grupo, 2 $\mathrm{mg}$ para el II grupo y $3 \mathrm{mg}$ para el III grupo por vía oral. Las lecturas de los grupos experimentales, se hicieron a los 3, 6 y 12 días, donde la concentración de creatinina para el grupo I fue de $0.3 \mathrm{mg} / \mathrm{dl}$, el grupo II 0.7 $\mathrm{mg} / \mathrm{dl} \mathrm{y}$ el grupo III $0.6 \mathrm{mg} / \mathrm{dl}$. La segunda lectura se realizó a los 6 días con el siguiente resultado: grupo I $0.4 \mathrm{mg} / \mathrm{dl}$, el grupo II 0.8 $\mathrm{mg} / \mathrm{dl}$ y el III con $0.9 \mathrm{mg} / \mathrm{dl}$. La tercera lectura se realizó el día 9: grupo I incremento a 0.6 $\mathrm{mg} / \mathrm{dl}$, el grupo II a $0.9 \mathrm{mg} / \mathrm{dl}$ y el III grupo $1.0 \mathrm{mg} / \mathrm{dl}$. Es decir que a medida que pasa el tiempo se aumenta los niveles de creatinina, lo que significa que el mercurio se acumula en los órganos dañándolos hasta generar complicaciones. Luego de 10 días de evaluaciones de las sintomatologías, se realizó las necropsias correspondientes con el $66.67 \%$ daño renal, $22.22 \%$ afecciones pulmonares y $11.11 \%$ de hemorragia interna. Estadísticamente no hubo diferencia significativa.

Palabras clave: hidrargiria, murinos,
\end{abstract} creatinina, mercurio.

\section{ABSTRACT}

The present research work aims to evaluate the pathophysiological effect of hydrargyria in murine systemic structures, which was carried out in the Laboratory of Physiology and Natural Sciences of the Faculty of Biological Sciences.

Twelve Mus musculus albino mice of the Balb / c variety were used, where treatments were carried out in three experimental groups and one control group. It is worth mentioning that 12 male and female mice, were used and the average age was 50 days. Each experimental group consisted of 3 mice, to which $1 \mathrm{ml}$ of mercury bichloride $1 \mathrm{mg}$ was applied for Group I, $2 \mathrm{mg}$ for Group II and $3 \mathrm{mg}$ for Group III by oral route. The readings of the experimental groups were made at 3,6 and 12 days, where the creatinine concentration for group I was $0.3 \mathrm{mg} / \mathrm{dl}$, group II $0.7 \mathrm{mg} / \mathrm{dl}$ and group III $0.6 \mathrm{mg} / \mathrm{dl}$. The second reading was performed at 6 days with the following result: group I 0.4 $\mathrm{mg} / \mathrm{dl}$, group II $0.8 \mathrm{mg} / \mathrm{dl}$ and III with 0.9 $\mathrm{mg} / \mathrm{dl}$. The third reading was made on day 9: group I increased to $0.6 \mathrm{mg}$ / $\mathrm{dl}$, group II to 0.9 $\mathrm{mg} / \mathrm{dl}$ and group III to $1.0 \mathrm{mg} / \mathrm{dl}$. In other words, as time goes by, creatinine levels increase, which means that mercury accumulates in the organs, damaging them until complications are generated. After 10 days of evaluating the symptoms, the corresponding autopsies were performed with $66.67 \%$ kidney damage, $22.22 \%$ pulmonary affections and $11.11 \%$ internal bleeding. Statistically there was no significant difference.

Key words: hydrargyria, murine, creatinine, mercury.

\section{RESUMO}

O presente trabalho de pesquisa visa avaliar o efeito fisiopatológico da hidrargiria em estruturas sistêmicas murinas, que foi realizado no Laboratório de Fisiologia e Ciências Naturais da Faculdade de Ciências Biológicas.

Foram utilizados 12 camundongos albinos Mus musculus da variedade Balb/c, onde os tratamentos foram realizados em três grupos experimentais e um grupo controle. Vale ressaltar que foram utilizados 12 camundongos machos e fêmeas e a idade média foi de 50 dias. Cada grupo experimental foi composto por 3 camundongos, aos quais foi aplicado $1 \mathrm{ml}$ de bicloreto de mercúrio $1 \mathrm{mg}$ para o Grupo I, $2 \mathrm{mg}$ para o Grupo II e 3 mg para o Grupo III por via oral. As leituras dos grupos experimentais foram feitas aos 3, 6 e 12 dias, onde a concentração de creatinina para o grupo I foi de $0,3 \mathrm{mg} / \mathrm{dl}$, grupo II 0,7 $\mathrm{mg} / \mathrm{dl}$ e grupo III $0,6 \mathrm{mg} / \mathrm{dl}$. A segunda leitura foi realizada aos 6 dias com o seguinte resultado: grupo I $0,4 \mathrm{mg} / \mathrm{dl}$, grupo II 0,8 $\mathrm{mg} / \mathrm{dl}$ e III com $0,9 \mathrm{mg} / \mathrm{dl}$. A terceira leitura foi feita no dia 9: grupo I aumentou para 0,6 $\mathrm{mg} / \mathrm{dl}$, grupo II para $0,9 \mathrm{mg} / \mathrm{dl}$ e grupo III para $1,0 \mathrm{mg} / \mathrm{dl}$. Ou seja, com o passar do tempo, os níveis de creatinina aumentam, o que significa que o mercúrio se acumula nos órgãos, danificando-os até que as complicações sejam geradas. Após 10 dias de avaliação dos sintomas, foram realizadas as autópsias correspondentes com $66,67 \%$ de lesão renal, $22,22 \%$ de afecções pulmonares e $11,11 \%$ de hemorragia interna. Estatisticamente não houve diferença significativa.

Palavras-chave: hidrargiria, murino, creatinina, mercúrio. 


\section{Introducción}

La hidrargiria, es una enfermedad provocada por el efecto del mercurio en el organismo viviente: planta, animal y especialmente en el humano, de actividad neurotóxica, con efectos adversos sobre la membrana celular y órganos internos. Dentro de los metales pesados tenemos: plomo, cadmio, cromo y mercurio, siendo una preocupación importante su toxicidad aguda y crónica, y su amplia variedad de fuentes de exposición.

Al determinar su significado biológico, se limita a su toxicidad, estos metales pesados ingresan al organismo viviente mediante las partículas o el aerosol presente el suelo, alimentos y el agua, las sales de mercurio son más peligrosas, por vía digestiva, respiratoria y la piel, posteriormente se acumulan en el riñón, hígado, el cerebro y la placenta.

Para la determinación del mercurio total existe una gran variedad de metodologías que proporcionan información parcial acerca de su impacto en la salud humana y el medio ambiente, como consecuencia se han desarrollado técnicas capaces de separar e identificar las diversas especies de mercurio para poder evaluar los distintos grados de impacto.

La presencia de mercurio está relacionada con el campo de la Salud Pública, donde la población de la región está en peligro de contactar directamente; las minas legales e ilegales, lavaderos de oros e indirectamente con diversos productos que contienen mercurio; en semillas con plaguicidas o biocidas y el agua contaminada de relaves mineros, desechos de estupefacientes, productos de belleza, materiales con mercurio deteriorados, entre otros.

El mercurio tiene múltiples usos como: cátodo en la electrólisis del cloruro sódico. Los compuestos químicos resultantes quedan contaminados con mercurio, su utilización en otras actividades industriales origina la contaminación de otros productos. El mercurio se emplea en la industria eléctrica, en instrumentos de control en el hogar y la industria, y en instrumental médico, amalgama odontológica y las prácticas de laboratorio.

Algunos agentes terapéuticos contienen mercurio inorgánico. En la extracción de oro se utilizan grandes cantidades de mercurio 
El vapor de mercurio emitido se convierte en formas solubles que son depositadas por la lluvia en el suelo y el agua. El tiempo de persistencia atmosférica para el vapor de mercurio es de hasta tres años, mientras que el de las formas solubles es de sólo algunas semanas. El cambio de especiación del mercurio desde las formas inorgánicas hasta las metiladas es la primera etapa del proceso de bioacumulación acuática. El metilmercurio ingresa en la cadena alimentaria de las especies productoras como consumidoras, generando su acumulación y las alteraciones correspondientes.

Por ello los metales pesados son considerados como cancerígenos por la Agencia de Investigación sobre el Cáncer de la OMS, a tomar conciencia sobre los posibles efectos perjudiciales del mercurio en la salud y el medio ambiente.

\section{Definir Objetivos.}

Evaluar el efecto fisopatológico de la hidrargiria en estructuras sistémicas de murinos.

Determinar los niveles de creatinina en los murinos afectados por la hidrargiria.

Observar el cuadro clínico en murinos con hidraragiria.

\section{Marco teórico}

\subsection{Antecedentes}

Bakir (1973), manifiesta que la absorción por parte del organismo humano de compuestos de mercurio orgánico provenientes de los alimentos es de aproximadamente el 7\% de la dosis ingerida, mientras que la absorción gastrointestinal de metil mercurio es prácticamente completa, la distribución del mercurio entre hematíes y plasma son los amas afectados. ${ }^{1}$

Cabañero (2005), señala que requiere disponer de una metodología analítica adecuada que combine un tratamiento de muestra que preserve las especies, una separación eficaz de las mismas y un sistema que permita su detección a los bajos niveles de concentración en que se encuentran habitualmente". ${ }^{2}$

Vinardell 2007), dice que la toxicología reguladora ha puesto cada vez mayor énfasis en la evaluación de los riesgos que presentan las sustancias químicas sobre la salud pública y ambiental, para lo cual realiza ensayos con animales. Debido a la presión de métodos alternativos a los tradicionales. puesta en el mercado de nuevos productos". 3 
Ruiz (2016), acota que el mercurio es un metal pesado con tiene efectos tóxicos, una de las vías de ingreso en los humanos es por el consumo de pescado, debido al riesgo representado para la salud, existe un gran interés en el desarrollo de técnicas analíticas sensibles y fiables para su determinación". ${ }^{4}$

Reyes (2016), plantean que “el problema específico de contaminación por mercurio $(\mathrm{Hg})$, Arsénico (As), Cadmio $(\mathrm{Cd})$ y Plomo $(\mathrm{Pb})$ en ambiente y alimentos. Se presenta una descripción sobre las fuentes de contaminación y exposición en seres vivos, así como la incorporación y retención en alimentos y productos de consumo humano". 5

Sarmiento (1999), señala que es un metal líquido a temperatura ambiente, que además de encontrarse en su estado elemental, se puede hallar como derivados inorgánicos y orgánicos. Es poco soluble y tóxico al ingerirse, puede emitir vapores a cualquier temperatura y ocasionar intoxicaciones. ${ }^{6}$

Osores (2010), manifiestan “que la contaminación del agua de los ríos por el mercurio constituye un grave problema de salud pública para la región, debido a las consecuencias de su difusión en la cadena trófica alimentaria y su llegada final a seres humanos, que pueden ocasionar intoxicación crónica inclusive intraútero; motivo por el cual embriones y fetos podrían ser contaminados de manera irreversible en forma temprana generando patologías neurológicas congénitas". ${ }^{7}$

Arriola (2013), señala que existen indicadores biológicos de exposición de estos metales suficientemente contrastados tanto en salud laboral como ambiental. Los niveles de plomo en sangre $(\mathrm{Pb}-\mathrm{S})$ y el daño que producen a nivel renal y la anemia. El cromo y el cadmio son considerados cancerígenos, el plomo y el mercurio presentan neurotoxicidad y el cromo es alergénico. El cadmio produce afectación pulmonar, renal, osteomalacia y osteoporosis. ${ }^{8}$

Ramírez. A (2008), concluye que el mercurio, metal pesado ampliamente utilizado por el hombre, es muy tóxico; produce daño al sistema nervioso central, perturbaciones del comportamiento y lesiones renales. ${ }^{9}$

\subsection{La hidrargiria.}

Las enfermedades o lesiones asociadas al mercurio se llaman hidrargirismo o mercurialismo e hidrargiria. La ingestión prolongada de alimentos contaminados con mercurio metílico provoca la enfermedad conocida como de Minamata. ${ }^{8}$

Se produce principalmente por ingestión o inhalación de cualquiera de las 3 formas de mercurio: elemental, orgánico e inorgánico. La exposición al mercurio 
elemental afecta a los pulmones (la inhalación de vapores de mercurio provoca tos, escalofríos, fiebre, dificultad respiratoria) y a la piel (leve inflamación, vesiculación, descamación, irritación, urticaria, eritema y dermatitis alérgica de contacto acompañada de dolor), así como al sistema nervioso periférico y central (depresión, paranoia, irritabilidad extrema, alucinaciones, incapacidad para concentrarse, pérdida de memoria, temblor de manos, cabeza, labios, lengua, mandíbula y párpados, pérdida de peso, baja temperatura corporal, somnolencia, cefaleas, insomnio, fatiga). La exposición al mercurio inorgánico provoca, por lo general, el desarrollo de un sabor metálico, dolor orofaríngeo localizado, náuseas, vómitos, diarrea sanguinolenta, dolor cólico abdominal, disfunción renal y anomalías neurológicas; mientras que el mercurio orgánico puede derivar en neurotoxicidad retardada. ${ }^{8}$

Intoxicación aguda. Los efectos inmediatos que puede producir el contacto con mercurio son:

a) Inhalación

- Escozor de garganta

- Dolor de cabeza

- Náuseas

- Pérdida del apetito y debilidad muscular

- Quemado o "refogado" de la amalgama

b) Contacto con ojos y piel:

- Enrojecimiento

- Irritación

- Amalgamación del mineral

c) Por ingestión:

- Vómitos,

- Diarrea

- Pérdida del apetito y

- Debilidad muscular

d) En caso de accidente los primeros auxilios a proporcionar son:

- En caso de inhalación: trasladar a la víctima al aire fresco. Buscar atención médica.

- En caso de contacto con la piel: quitar la ropa contaminada. Lavar el área afectada con agua y jabón. Buscar atención médica. 
- En caso de contacto con los ojos: lavar los ojos inmediatamente con agua. Buscar atención médica.

- En caso de ingestión: enjuagar la boca con agua. Buscar atención médica.

Intoxicación crónica. La exposición prolongada o repetida a Mercurio puede provocar sobre todo lesiones en:

- Riñones,

- Cerebro y el

- Sistema nervioso.

Mercurio. Es un mineral que se encuentra en tres estados: metálico $(\mathrm{Hg} 0)$, mercurioso $\left(\mathrm{Hg}^{++}\right)$y mercúrico $\left(\mathrm{Hg}^{++}\right)$. También forman otros compuestos organometálicos, algunos de los cuales tienen usos industriales y agrícola. El mercurio elemental tiene una presión de vapor sumamente elevada. La atmósfera saturada a $20{ }^{\circ} \mathrm{C}$ tiene una concentración más de 200 veces superior a la de la concentración comúnmente aceptada para la exposición profesional.

El mercurio elemental y los haluros de compuestos alquil mercuriales son solubles en disolventes no polares. El vapor de mercurio es más soluble en plasma, sangre entera y hemoglobina que en agua destilada, donde sólo se disuelve ligeramente. Los compuestos organometálicos son estables, aunque algunos son fácilmente descompuestos por los organismos vivos. ${ }^{10}$

Cinética y metabolismo. Los resultados de los estudios realizados tanto en personas como en animales indican que alrededor del $80 \%$ del vapor de mercurio metálico inhalado es retenido por el organismo, mientras que el mercurio metálico líquido se absorbe mal en el tracto gastrointestinal (menos del 1\%).

Los aerosoles de mercurio inorgánico inhalados se depositan en el tracto respiratorio y son absorbidos a una velocidad que depende del tamaño de las partículas.

Pueden producirse varias formas de transformación metabólica:

- $\quad$ oxidación del mercurio metálico a mercurio bivalente;

- $\quad$ reducción del mercurio bivalente a mercurio metálico;

- metilación del mercurio inorgánico;

- conversión del metilmercurio en mercurio inorgánico bivalente.

La oxidación de vapor de mercurio metálico a mercurio iónico bivalente no es lo bastante rápida como para impedir el paso de mercurio elemental a través de la barrera hematoencefálica, la placenta y otros tejidos. La oxidación en esos tejidos sirve como 
filtro para retener el mercurio y lleva a su acumulación en el cerebro y los tejidos fetales.

Valores normales. La limitada información de que se dispone sobre mineros fallecidos muestra la existencia de concentraciones de mercurio en el cerebro de varios $\mathrm{mg} / \mathrm{kg}$, años después de finalizar la exposición, con valores aún más altos en algunas partes del cerebro. No obstante, la falta de control de la calidad en el análisis hace inciertos estos datos. Entre un pequeño número de dentistas fallecidos, sin síntomas conocidos de intoxicación por mercurio, los niveles de éste variaron desde concentraciones muy bajas hasta varios cientos de $\mu \mathrm{g} / \mathrm{kg}$ en la corteza del lóbulo occipital y desde unos $100 \mu \mathrm{g} / \mathrm{kg}$ hasta unos cuantos $\mathrm{mg} / \mathrm{kg}$ en la hipófisis.

Los niveles de mercurio en la orina y la sangre pueden usarse como indicadores de la exposición, siempre que ésta sea relativamente constante a largo plazo y se evalúe en un grupo. Los datos de exposición recientes son más fiables que los que se citan en Criterios de Salud Ambiental 1.

WHO (1978). Se observan niveles en la orina de unos $50 \mu \mathrm{g} / \mathrm{g}$ de creatinina tras la exposición profesional a unos $40 \mu \mathrm{g}$ de mercurio por $\mathrm{m} 3$ de aire. Esta relación $(5: 4)$ entre orina y niveles atmosféricos es mucho más baja que la de 3:1 estimada por la WHO. ${ }^{11}$

La diferencia puede deberse en parte a la distinta técnica de muestreo para evaluar la exposición atmosférica. Una exposición de $40 \mu \mathrm{g}$ de mercurio/m3 de aire corresponderá a unos 15-20 $\mu \mathrm{g}$ de mercurio/litro de sangre. Sin embargo, la interferencia debida a la exposición al metilmercurio puede hacer más difícil evaluar la exposición a bajas concentraciones de mercurio inorgánico por medio de análisis de sangre. Una forma de salvar esos problemas es analizar el mercurio en el plasma o analizar tanto el mercurio inorgánico como el metilmercurio. El problema de la interferencia debida al metilmercurio es mucho menor cuando se analiza la orina, puesto que el metilmercurio se excreta con la orina en grado sumamente reducido. ${ }^{7}$

\subsection{Efectos en el hombre}

Se dispone de muy poca información sobre los niveles cerebrales de mercurio en los casos de envenenamiento, y no se sabe nada que permita estimar una concentración carente de efectos observados o una curva dosis/respuesta.

Aunque la exposición de la población general es por lo general reducida, en ocasiones puede elevarse hasta el nivel de exposición profesional y puede incluso llegar 
a ser tóxica. Así, la manipulación incorrecta de mercurio líquido ha dado origen a casos graves de intoxicación.

Arriola (2013). Aunque muchos de los metales pesados analizados se encuentran en forma natural en el ambiente, y algunos son elementos esenciales para la salud humana, hay otros elementos que pueden causar graves problemas en la salud de las personas. ${ }^{8}$

Los efectos adversos a la salud asociados con la exposición a los metales pesados, pueden limitarse con la con la implementación de intervenciones adecuadas.

Los niveles de estos metales pesados analizados en sangre, orina de mujeres en edad fértil y de niños probablemente reflejan las exposiciones a las vías como el agua, suelo, y los alimentos.

Tabla 1. Descripción del número de análisis y su concentración de mercurio según sexo.

\begin{tabular}{ccccc} 
SEXO & LOD- 20,00 & $\mathbf{2 0 , 0 1 - 2 0 0 , 0 0}$ & $\mathbf{2 0 0 , 0 1 - 3 2 7 1 , 5 7}$ & TOTAL \\
\hline Femenino & 19 & 2 & 1 & 22 \\
Masculino & 24 & 1 & 0 & 25 \\
No indica & 11 & 0 & 0 & 11 \\
Total & 54 & 3 & 1 & 58 \\
\hline
\end{tabular}

WHO (1978), propone los niveles de mercurio en la orina y la sangre pueden usarse como indicadores de la exposición, siempre que ésta sea relativamente constante a largo plazo y se evalúe en un grupo. Los datos de exposición recientes son más fiables que los que se citan en Criterios de Salud Ambiental.

Se observan niveles en la orina de unos $50 \mu \mathrm{g} / \mathrm{g}$ de creatinina tras la exposición profesional a unos $40 \mu \mathrm{g}$ de mercurio por $\mathrm{m}^{3}$ de aire. ${ }^{11}$

Una forma de salvar esos problemas es analizar el mercurio en el plasma o analizar tanto el mercurio inorgánico como el metilmercurio. El problema de la interferencia debida al metilmercurio es mucho menor cuando se analiza la orina, puesto que el metilmercurio se excreta con la orina en grado sumamente reducido. ${ }^{11}$

Tirado (2015). En la sociedad del siglo XXI enfocada hacia el desarrollo socioeconómico, la continua industrialización, la práctica de la minería y de actividades productivas como la agricultura resultan ser opciones atractivas; sin embargo, en el desarrollo de estas actividades, es frecuente el uso y la emisión de algunos químicos hacia el medio ambiente, entre los cuales se encuentran los metales pesados. ${ }^{12}$ 
Tabla 2. Valores de referencia de algunos metales pesados (cadmio, mercurio, zinc, cobre, plomo).

\begin{tabular}{|c|c|c|c|c|c|} 
Metales & Uñas & Cabello & Saliva & Orina & Sangre \\
\hline Cadmio & $0,018 \mathrm{ug} / \mathrm{g}$ & $0,41 \mathrm{ug} / \mathrm{g}$ & $0,43 \mathrm{ug} / \mathrm{g}$ & $0,32 \mathrm{~g} / \mathrm{L}$ & $0,55 \mathrm{ug} / \mathrm{g}$ \\
\hline Mercurio & $0,033 \mathrm{ug} / \mathrm{g}$ & $0,037 \mathrm{ug} / \mathrm{g}$ & $0,015 \mathrm{mg} / \mathrm{g}$ & $0,89 \mathrm{~g} / \mathrm{L}$ & $0,95 \mathrm{~g} / \mathrm{L}$ \\
\hline Plomo & $0,062 \mathrm{ug} / \mathrm{g}$ & $4,06 \mathrm{ug} / \mathrm{g}$ & $25,5 \mathrm{ug} / \mathrm{g}$ & $1,63 \mathrm{~g} / \mathrm{L}$ & $56,8 \mathrm{mg} / \mathrm{L}$ \\
\hline Zinc & $129 \mathrm{ug} / \mathrm{g}$ & $102 \mathrm{ug} / \mathrm{g}$ & $23 \mathrm{mg} / \mathrm{g}$ & $0,5 \mathrm{mg} / \mathrm{g}$ & $0,70 \mathrm{mg} / \mathrm{L}$ \\
\hline Cobre & $65,8 \mathrm{ug} / \mathrm{g}$ & $5,5 \mathrm{ug} / \mathrm{g}$ & $16,9 \mathrm{ug} / \mathrm{g}$ & $11,38 \mathrm{ug} / \mathrm{L}$ & $0,75 \mathrm{mg} / \mathrm{L}$ \\
\hline
\end{tabular}

En el organismo de los seres vivos, hay pequeñas cantidades de algunos metales pesados que pueden intervenir en reacciones bioquímicas, pero otros metales no representan utilidad biológica. Por esto, la exposición ocupacional y la exposición pasiva a metales pesados presentes en el ambiente o que provienen de la dieta deben ser reguladas, ya que cantidades superiores a las consideradas normales pueden ocasionar ciertas enfermedades. El control de la exposición a metales pesados incluye monitorear los niveles presentes en distintas matrices biológicas como la sangre, la orina, la saliva, el cabello y las uñas. Cada una de estas matrices estará indicada según el tipo de exposición que ha experimentado el individuo; además, ofrecerá algunas ventajas y limitaciones. Es por eso que la presente revisión pretende informar sobre los efectos ocasionados en el organismo por niveles excesivos de algunos metales pesados y la utilidad que ofrecen las matrices biológicas en la determinación de estos niveles.

Reyes. Y (2016). Los límites máximos permisibles de concentración de metales están muy bien establecidos en agua. Sin embargo, aún falta por definir los límites de concentración y riesgo de $\mathrm{Hg}$, As y Cd en hortalizas, legumbres y cereales, para permitir estandarizar y consensuar los estudios de contaminación que se están realizando, así como los efectos tóxicos y en ambiente. ${ }^{5}$

Se recomienda realizar monitoreo y detección de contaminantes en agua, fauna y flora, para trazar mapas de concentración de estos contaminantes y favorecer los planes de acción conducentes a tareas de mitigación y remediación. Es importante atender puntos estratégicos de Colombia, como el rio Bogotá y el Lago de Tota, entre otros. ${ }^{5}$

Es necesario realizar estudios sistemáticos, científicos y complementarios del contenido de metales pesados en diferentes matrices que permitan la elaboración de informes fehacientes y con visión del futuro sobre el estado del rio Bogotá y el Lago de Tota e incidir directamente sobre la calidad y la localización, con ello se puede facilitar una mejor identificación y control de dichas afluencias para que los organismos pertinentes puedan 
tomar medidas preventivas y decisiones de mejora. El análisis de especiación proporciona información respecto a la biodisponibilidad en unas determinadas condiciones medioambientales y pequeños cambios en los parámetros fisicoquímicos del medio. ${ }^{5}$ 
Tabla 3. Límites máximos permisibles de concentración de metales pesados $(\mathrm{Hg}$, $\mathrm{As}, \mathrm{Cd}, \mathrm{y} \mathrm{Pb}$ ) en agua y suelo y alimentos humanos.

\begin{tabular}{ccccc} 
Agua mg/L & $\mathbf{H g}$ & $\mathbf{A s}$ & $\mathbf{C d}$ & $\mathbf{P b}$ \\
\hline Consumo humano & 0,001 & 0,05 & 0,01 & 0,05 \\
Marina y estuarios & 0,0001 & 0,05 & 0,05 & 0,01 \\
Uso agrícola & 0,001 & 0,1 & 0,01 & 0,05 \\
Uso pecuario & 0,01 & 0,2 & 0,05 & 0,05
\end{tabular}

La contaminación ambiental se posiciona como uno de los más importantes problemas que afectan a la sociedad del siglo XXI. La pérdida de calidad del aire, del recurso hídrico y de suelos disponibles para actividades agrícolas se ha incrementado exponencialmente. La tasa de contaminación del agua puede ser estimada en 2000 millones de metros cúbicos diarios.

Se hace evidente una crisis de este recurso para los próximos años, lo que podría comprometer el cumplimiento de uno de los objetivos de Desarrollo del Milenio de la Organización de Naciones Unidas (ONU-DAES, 2005-2015). En septiembre de 2015, la Asamblea general de la ONU, acordó como objetivo: "asegurar la disponibilidad y la gestión sostenible de agua y saneamiento para todos", otorgándole al agua un carácter prioritario para todos los países miembro. Cabe destacar que el año 2015 fue crítico para la agenda en materia de agua y saneamiento. ${ }^{15}$

Por su elevada toxicidad, el impacto causado en salud por exposición prolongada o por bio acumulación de metales pesados resulta alarmante. Dependiendo del tipo de metal o metaloide, se producen afecciones que van desde daños en órganos vitales hasta desarrollos cancerígenos. ${ }^{13,14}$

A nivel global, se han reportado casos que dan cuenta de las afecciones en la salud por causa del consumo de alimentos contaminados por metales pesados. Sánchez, et al (2010), afirma que un caso relevante ocurrió en Japón en la década de los cincuenta, en donde la población ubicada en las riberas del río Jintsu, aguas abajo de una zona minera de zinc $(\mathrm{Zn})$, plomo $(\mathrm{Pb})$ y cobre $(\mathrm{Cu})$, se vio afectada por el consumo de arroz proveniente de cultivos contaminados con cadmio $(\mathrm{Cd})$ procedente de los vertimientos de las minas. Esta ingesta produjo una enfermedad conocida como Itai Itai o osteoartrítis la cual afecta principalmente el tejido óseo. ${ }^{16}$

De otra parte, en la población infantil de Torreón, Coahuila ubicada en Norte y centro de México se han reportado casos por envenenamiento principalmente por plomo 
$(\mathrm{Pb})$ proveniente de actividades industriales que incorporan este metal a la cadena alimenticia y al agua.

Arnous et al (2015) señala que la OMS y algunas autoridades ambientales, han establecido niveles de riesgo en función de la concentración de metales en aguas de consumo humano y alimentos. Los mapas realizados en algunas regiones del mundo muestran un importante incremento en la concentración por encima de los límites establecidos, que las clasifica como de alto riesgo. ${ }^{17}$

Gonzales et al (2015), afirma para el caso del arsénico, la población en riesgo de exposición supera los 150 millones, esto obliga a fortalecer los programas de saneamiento apoyados en tecnologías emergentes como la bio y nanotecnología para el desarrollo de procesos y estrategias experimentales en tareas de detección, cuantificación y remediación. ${ }^{18}$

Ramírez, A. (2008). En los últimos 50 años, con la aparición de nuevas sustancias químicas, si bien es verdad punto de apoyo en el avance industrial, el panorama se complica y no es menos cierto que este avance ha producido empeoramiento de las condiciones de higiene y salud en el trabajo, que literalmente agreden al trabajador. Es pues imprescindible conocer la naturaleza y acción biológica de cada agente, para controlarlo con exámenes médico ocupacionales de exposición. El uso de biomarcadores en exposición a metales pesados tiene una finalidad práctica. ${ }^{9}$

Osores, F. (2016). Se ha utilizado un proceso de búsqueda sistemática a fin de consolidar evidencia científica que permita aproximar un diagnostico multidimensional de salud ambiental en la provincia de Espinar asociado a sus actividades antropogénicas, con un nivel de certeza que alcance la aplicación de los principios protectivo y preventivo en ambiente y salud. ${ }^{7}$

Se realizaron combinaciones como: "human healht and mining" con 6047 resultados, "communities health and mining" con 466 resultados, "mining and cáncer" con 3583 resultados, "non-occupational exposure and mining" con 21 resultados, "heavy metals and mining and human healht" con 1235 resultados y así sucesivamente. Se ha tomado en cuenta una recopilación informativa y su sistematización, en el ámbito de la Micro-Red de Salud de Espinar que es parte de la Red Canas Canchis Espinar, tomando como información base, los estudios de monitoreo de exposición a metales pesados realizados en la zona tanto el 2010 como el 2013 por el Centro de Salud Ocupacional y Protección del Ambiente para la Salud (CENSOPAS), centro adscrito como al Instituto Nacional de Salud (INS), organismo público descentralizado del 
Ministerio de Salud del Perú (MINSA). También, se han revisado distintos monitoreos ambientales realizados en el ámbito de nuestra revisión sistemática. ${ }^{19}$

Se siguió una estrategia de búsqueda sistemática en base a lo propuesto por la Pirámide de Haynes. Esta permite ubicar los niveles de evidencia y en cuyo vértice encontramos los sistemas de soporte del ordenador, que son en esencia los componentes de la realidad que priman hipotéticamente en ese momento. En Medicina Clínica Ambiental y Toxicológica, la mayor parte de los estudios generadores de evidencia se encuentran distribuidos entre las secciones bajas e intermedia de la pirámide.

\subsection{Efectos en animales}

Cabañero. A (2005), el método analítico desarrollado basado en el secado previo de la muestra en estufa y el empleo posterior de $\mathrm{HCl}$ como medio extractante permite la extracción de las especies de mercurio presentes en muestras biológicas de forma cuantitativa, manteniendo su integridad durante todo el proceso de extracción. Además, ha demostrado que los extractos ácidos son estables durante una semana, no produciéndose variaciones en la concentración de mercurio total. El almacenamiento de los extractos durante el periodo mencionado, evitándose la necesidad del análisis inmediato. $^{2}$

El método ha sido aplicado satisfactoriamente para la determinación de las especies de mercurio en pescados, y validado mediante su aplicación a un material de referencia. La metodología desarrollada permite la determinación de las especies de mercurio sin necesidad de emplear procedimientos más laboriosos como derivación por etilación.

El método desarrollado basado en la combinación de la energía procedente de una sonda de ultrasonidos y un tratamiento enzimático permite la extracción del contenido total de selenio y sus especies en muestras biológicas en un tiempo muy inferior al requerido por otros procedimientos, sin que se originen pérdidas ni transformaciones de las especies químicas estudiadas.

Este método de extracción ha sido aplicado satisfactoriamente a muestras de origen vegetal y animal y se caracteriza por el bajo consumo de reactivos, la simplicidad y seguridad del procedimiento.

La determinación de $\mathrm{Hg}$ total y sus especies en pescados de elevado consumo en España y Portugal pone de manifiesto que los pescados seleccionados tienen la habilidad de acumular elevadas concentraciones de $\mathrm{Hg}$, fundamentalmente $\mathrm{MeHg}$. 
Tabla 4. Ingesta media diaria y evaluación de ingesta de mercurio por la población en general.

\begin{tabular}{r|lll} 
Fuente exposición & Hg vapor & Hg inorgánico & Metilmercurio \\
\hline Aire & 0.030 & 0.002 & 0.008 \\
Pescado & 0.024 & 0.600 & 2.4 \\
Otros & 0 & 3.6 & 0 \\
Agua potable & 0 & 0.050 & 0 \\
Amalgama dental & 3.8 & 0 & 0 \\
Total & 4.34 & 4.252 & 2.408
\end{tabular}

Estudios de bioaccesibilidad de $\mathrm{Hg}$ y Se llevados a cabo mediante la simulación de una digestión gastrointestinal in vitro demostraron que ésta depende del tipo de muestra analizada, excepto en el caso de la Se/Met, lo que implica que esta especie se encuentra presente en los pescados en proteínas de la misma naturaleza. Las especies de $\mathrm{Hg}$ y Se permanecen inalteradas durante todo el proceso de digestión, resultando más disponible las especies de Se.

El pescado más recomendable para el consumo humano puede calcular la relación molar Selenio/Mercurio. De todos los pescados estudiados la sardina ha resultado ser la especie más idónea.

El enriquecimiento de piensos con un agente antagónico del efecto tóxico del mercurio y selenio y con materiales adsorbentes (bentonita y sepiolita) ha resultado ser adecuado para reducir la toxicidad del Hg inorgánico en aves. Además, el empleo de bentonita en la dieta puede ser beneficioso en caso de que los animales consuman alimentos contaminados con $\mathrm{MeHg}$, ya que disminuye su acumulación.

La cuantificación, especiación y distribución de los compuestos de $\mathrm{Hg}$ y Se, pone de manifiesto la biotransformación de ambos elementos, así como el antagonismo ejercido por el Se como resultado de su interacción con el $\mathrm{Hg}$ cuando se administran simultáneamente en aves. ${ }^{2}$

Escobar, O (2010). Llega a la conclusión Los niveles de mercurio bioacumulados en los depredadores analizados permitieron diferenciar entre los grupos de depredadores, posicionando al grupo tiburones, con excepción de $S$. zygaena, como los depredadores con las mayores bioacumulaciones de mercurio con respecto a las especies afines a la pesca deportiva. ${ }^{20}$

A pesar de registrar valores altos de mercurio en algunos tiburones, la escasa correlación entre las longitudes y las bioacumulaciones de mercurio, así como los niveles de selenio encontrados, pueden ser evidencia de los mecanismos de desintoxicación que poseen los animales marinos. El patrón de bioacumulación de 
mercurio observado en los depredadores en la costa occidental de Baja California Sur, no fue similar al presentado en el Golfo de California por otros autores para las mismas especies. Por lo que, en la zona del Golfo de California, existen más fuentes potenciales de contaminación provenientes de las actividades de las poblaciones aledañas (agricultura, industria eléctrica, etc.). ${ }^{20}$

Tabla 5. Proporción molar de las concentraciones de mercurio y selenio $(\mathrm{Hg} / \mathrm{Se})$ en músculo de las diferentes especies de peces pelágicos que se capturan en Baja California.

\begin{tabular}{cccc}
\hline Especie & umol Hg/g & umol Se/g & Hg/Se \\
\hline Coryphaena hyppurus & 1.15 & & \\
Spirna zigaena & 0.80 & & \\
Piranaceae glauca & 6.93 & 2.53 & \\
Isurus oxyrinchus & 5.53 & 4.31 & \\
Alopias pelágicus & 7.03 & 1.27 & \\
Carcharinus limbatus & 18.84 & 1.77 & \\
Thunnus albacares & 0.70 & & \\
Makaira nigricans & 21.84 & & \\
Kajiura audax & 1.60 & & \\
\end{tabular}

Debido a que los mayores niveles de mercurio se presentaron en el músculo y no en las aletas de los tiburones, se deduce que las bioacumulaciones pueden estar influidas por la cantidad de lípidos del músculo, debido a las características lipofílicas del mercurio.

Algunas especies de cefalópodos analizados no bioacumulan niveles altos de mercurio, por lo que no aportan cantidades considerables a los depredadores; sin embargo, al presentar niveles menores de mercurio que sus depredadores, es un reflejo de que los niveles tróficos altos poseen una mayor cantidad de mercurio.

Esta contaminación, proviene, entre otros causales, del uso para riego de aguas afectadas. De igual manera, se han encontrado metales en diferentes concentraciones en peces, carnes y leche resultado de la bio-acumulación y movilidad desde el ambiente a las fuentes hídricas.

Vinardell, M. (2007). Los ensayos de irritación ocular consisten en la administración de los productos directamente en el ojo de un conejo, que es el animal de elección. ${ }^{3}$ 
York (1998), aplica el método tradicional de estudio de la irritación y la corrosión ocular in vivo se ha venido realizando mediante el denominado "ensayo de Draize" (1994), con muchos detractores debido a su agresividad, a su subjetividad y a la falta de correlación con los efectos reales en humanos. ${ }^{21}$

Una variante del ensayo es la determinación de irritación dérmica en la piel del conejo luego de ser rasurada, que también resulta muy subjetiva, ya que la valoración de las lesiones ocasionadas es realizada por un experimentador

enrojecimiento provocado en la piel después de la aplicación del producto en estudio. Los ensayos de sensibilización mediante el método de maximización realizado en cobayos son los más utilizados.

Ruiz, I. (2016). El mercurio es un metal pesado con tiene efectos tóxicos, una de las vías de ingreso en los humanos es por el consumo de pescado, debido al riesgo representado para la salud, existe un gran interés en el desarrollo de técnicas analíticas sensibles y fiables para su determinación. Para la determinación del mercurio total existe una gran variedad de metodologías que proporcionan información parcial acerca de su impacto en la salud humana y el medio ambiente, como consecuencia se han desarrollado técnicas capaces de separar e identificar las diversas especies de mercurio para poder evaluar los distintos grados de impacto. Se realiza una propuesta del principio básico, ventajas e inconvenientes de las principales técnicas en la determinación de mercurio total y metilmercurio en matrices alimentarias (músculo de pescado) empleadas por métodos analíticos directos e indirectos, tales como: la espectrometría de absorción atómica de vapor frío (CV AAS), vapor frío espectrometría de fluorescencia atómica (CV AFS), plasma acoplado inductivamente-espectrometría de masas (ICP-MS) y analizador de mercurio. También se presenta un breve texto con el uso de técnicas cromatográficas para el análisis de especiación del mercurio orgánico. Se han desarrollado muchas técnicas analíticas para determinar el mercurio, las cuales han logrado tener un mayor grado de especificidad y selectividad con equipos muy sensibles, para determinar concentraciones en el de nivel ultratrazas y lograr la identificación y separación de los diferentes compuestos del mercurio, para garantizar el riesgo asociado al consumo de pescado. ${ }^{4}$

Vega, M. (2002). El ratón viene ha ser utilizado en los experimentos e investigaciones biomédicas. Pertenece al orden Rodentia, género Mus y especie, Mus musculus, el ratón blanco Suiss o SPF, es una variedad albina, es inofensivo, pero a veces muerde con fiereza sino guarda las precauciones necesarias. Es de gran 
prolificidad y gran adaptación, con amplia variedad genética y sensibilidad para determinados agentes infecciosos, es modelo representativo de reactivos biológicos y tóxicos. Actualmente cuenta con estirpes y líneas nuevas. ${ }^{22}$

La aplicación actual de animales de experimentación con mercurio en los roedores, presentarán patologías infecciosas en el tracto respiratorio, intestinal e hígado, la cual puede conllevar a la mortalidad y morbilidad.

El riñón es el depósito principal de mercurio tras la administración de vapor de mercurio elemental o de compuestos de mercurio inorgánico $(50-90 \%$ de la carga corporal de los animales). De modo significativo, más mercurio es transportado al cerebro del ratón y el mono tras la inhalación de mercurio elemental que tras la inyección intra- venosa de dosis equivalentes de la forma mercúrica. El cociente hematíes/plasma en el hombre es mayor $(>1)$ tras la administración de mercurio elemental que tras la de mercurio mercúrico, y la proporción de mercurio que atraviesa la barrera placentaria es mayor. Sólo una pequeña fracción del mercurio bivalente administrado ingresa en el feto de los roedores.

La reducción del mercurio bivalente a $\mathrm{Hg} 0$ se ha demostrado tanto en animales (ratones y ratas) como en el hombre. La descomposición de los compuestos organomercuriales, incluido el metilmercurio, es también una fuente de mercurio mercúrico.

\subsection{Efecto en las plantas.}

Huang et al (2014) señala que específicamente, la contaminación del agua por metales pesados ocasionada por vía antrópica y natural, está afectando drásticamente la seguridad alimentaria y salud pública. ${ }^{23}$

Singh et al (2010), manifiesta que estudios recientes reportan la presencia de metales pesados y metaloides tales como mercurio $(\mathrm{Hg})$, arsénico (As), plomo $(\mathrm{Pb})$, cadmio $(\mathrm{Cd})$, zinc $(\mathrm{Zn})$, níquel $(\mathrm{Ni})$ y cromo $(\mathrm{Cr})$ en hortalizas tales como la lechuga, repollo, calabaza, brócoli y papa. ${ }^{24}$

\section{Materiales y métodos}

\subsection{Ubicación de la zona de estudio}

\subsubsection{Ubicación Política}


El presente trabajo de investigación se desarrolla en el distrito de Ayacucho, en las instalaciones del laboratorio de Fisiología y Ciencias Naturales perteneciente a la Facultad de Ciencias Biológicas de la UNSCH.

\subsubsection{Ubicación geográfica}

El distrito de Ayacucho se encuentra ubicado en la región centro sur de los andes peruanos, cuyas coordenadas son: latitud sur $13^{\circ} 09^{\prime} 26^{\prime \prime}$ y longitud oeste $74^{\circ} 13^{\prime} 22^{\prime \prime}$ del meridiano de Greenwich; a una altitud de 2,747 msnm.

\subsection{Población y muestra}

\subsubsection{Población}

La población en estudio comprende todos los ratones albinos que se encuentran en la ciudad de Ayacucho.

\subsubsection{Muestra}

La muestra está constituida por 12 ratones albinos Mus musculus de la variedad Balb, establecidos en jaulas metabólicas especiales. Alimentación ad libitum, la longitud de la nariz a la cola fue de $15 \mathrm{~cm}$.

\subsection{Método de la Creatinina de Wiener. ${ }^{25}$}

\subsubsection{Fundamento.}

La creatinina reacciona con el picrato alcalino denominado reacción de Jaffe, produciendo un cromógeno rojo, la velocidad de reacción, bajo condiciones controladas, es una medida de concentración de creatinina de la muestra, puesto que se comporta como una reacción cinética de primer orden para la creatinina, por otra parte, se ha demostrado que, entre los 30 segundos y los 5 minutos posteriores al inicio de la reacción, el incremento de color se debe exclusivamente a la creatinina, que nos indicara el daño renal producido.

\subsubsection{Recolección y estabilidad de la muestra.}

La muestra es recolectada en tubos heparinizados (monojet tapón verde) o tubos metal free con EDTA (tapón azul marino). El volumen mínimo de muestra es un mililitro de sangre entera que contenga $1.5 \mathrm{ul}$ de heparina de $5000 \mathrm{UI}$ o $1.5 \mathrm{mg}$ de EDTA como anticoagulante. La sangre entera se puede conservar a $4^{\circ} \mathrm{C}$ aproximadamente por una semana.

\subsubsection{Materiales requeridos.}

Espectrofotómetro capaz de leer a $500 \pm 10 \mathrm{~nm}$. 
Micropipetas y pipetas para medir volúmenes indicados.

Cubetas espectrofotométricas

Baño de agua a $25^{\circ} \mathrm{C}$

Cronometro

Bicloruro de Mercurio $\left(\mathrm{HgCl}_{2}\right)$

\subsubsection{Preparación de muestras biológicas.}

Los 3 ratones se agruparon en 3 grupos experimentales y 1 grupo control. Sumando un total de 12 ratones albinos.
A. aplicar $1.0 \mathrm{ml}$ de la solución de mercurio $(1 \mathrm{mg} \mathrm{Hg} / \mathrm{kg} /$ día), vía oral por 6 días a 3 ratones.
B. aplicar $1.0 \mathrm{ml}$ de la solución de mercurio $(2 \mathrm{mg} \mathrm{Hg} / \mathrm{kg} /$ día $)$, vía oral por 6 días a 3 ratones.
C. aplicar $1.0 \mathrm{ml}$ de la solución de mercurio $(3 \mathrm{mg} \mathrm{Hg} / \mathrm{kg} /$ día), vía oral por 6 días a 3 ratones.
D. grupo control aplicar $1 \mathrm{ml}$ del placebo (solución salina fisiológica) como testigo a 3 ratones.

\subsubsection{Análisis de las muestras según el Método de Wiener. ${ }^{25}$}

Procedimiento para la técnica de suero o plasma. Se realizaron tres lecturas al 3, 6 y el 9 día.

- Llevar el aparato a cero con agua destilada. En dos cubetas espectrofotométricas marcadas S (Standar) y D (Desconocido).

- A la cubeta $\mathrm{S}$ colocar 1,2 $\mathrm{ml}$ del reactivo de trabajo y $0,2 \mathrm{ml}$ del estándar.

- A la cubeta D agregar 1,2 $\mathrm{ml}$ del reactivo de trabajo y $0,2 \mathrm{ml}$ de la muestra

- Mezclar inmediatamente, marcar el cronómetro y proseguir la incubación. A los 30 segundos medir la absorbancia de S1 y D1 y continuar la incubación. Medir nuevamente la absorbancia S2 y D2 a los 5 minutos, después de la primera lectura.

\subsubsection{Cálculos de los resultados.}

Creatinina en suero $(\mathrm{mg} / \mathrm{dl})=(\mathrm{D} 2-\mathrm{D} 1) \times \mathrm{F}$

$\mathrm{F}=\underline{20 \mathrm{mg} / \mathrm{l}}$ 
3.3.8. Diagnóstico de la sintomatología y la necropsia. Lo cual se realizó el decimo día a todos los ratones en experimentación.

\subsection{Análisis de datos}

Los datos obtenidos en el estudio serán traspasados a Excel con el fin de visualizarlos para su análisis estadístico. Se utilizará tablas de contingencia acompañados de gráficos para una mejor comprensión de los resultados y se usarán las pruebas de Intervalo de confianza para hallar la relación existente entre dos variables mediante la significación estadística, con un nivel de confianza del 95\%. y el análisis postural se realizará por medio del software SSPS v20.

\section{Resultados}

Tabla 6. Promedios de los niveles de creatinina $\mathrm{mg} / \mathrm{dl}$ debido al efecto patológico de la hidrargiria en murinos.

\begin{tabular}{c|ccc} 
TRATAMIENTOS & 3 DÍAS & 6 DÍAS & 9 DÍAS \\
\hline CONTROL & 0 & 0 & 0 \\
I & 0,3 & 0,7 & 0,6 \\
II & 0,4 & 0,8 & 0,9 \\
III & 0,6 & 0,9 & 1
\end{tabular}

Figura 1. Niveles de creatinina $\mathrm{mg} / \mathrm{dl}$ resultado del efecto patológico de la hidrargiria en murinos.

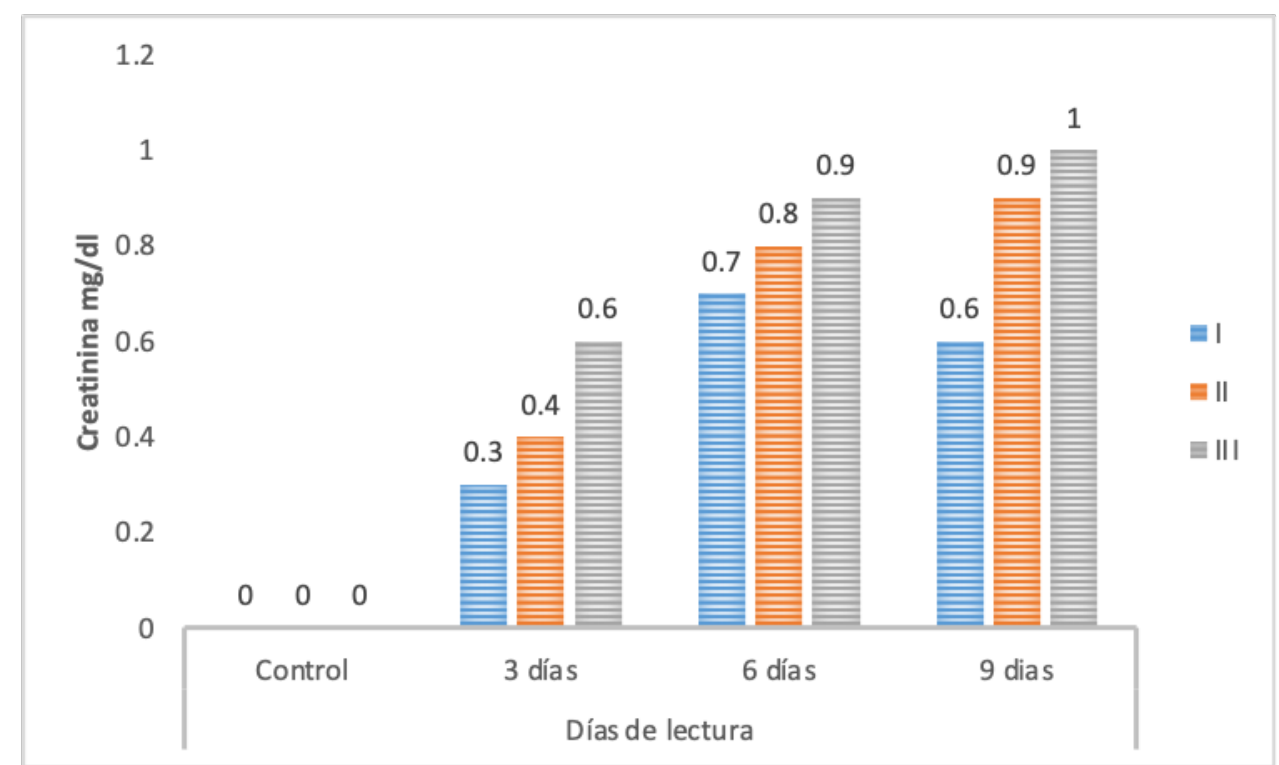


Tabla 7. Sintomatología patológica observada en el grupo experimental de murinos con hidrargiria.

\begin{tabular}{r|ll} 
SÍNTOMAS & $\mathrm{N}$ & $\%$ \\
\hline Edema & 4 & 44,45 \\
Alterac. Neurolog. & 3 & 33,33 \\
Decolor. Piel & 1 & 22,22 \\
Total & 9 & 100
\end{tabular}

Tabla 8. Necropsia realizada en la fisiología sistémica del grupo experimental de murinos afectados por la hidrargiria.

\begin{tabular}{l|ll} 
NECROPSIA & N & \% \\
\hline DAÑO RENAL & 6 & 66,67 \\
AFECCIÓN & 2 & 22,22 \\
$\begin{array}{l}\text { PULMONAR } \\
\text { HEMORRAGIAS }\end{array}$ & 1 & 11,11 \\
TOTAL & 9 & 100
\end{tabular}

\section{Discusión}

Lecturas realizadas de los niveles de creatinina $\mathrm{mg} / \mathrm{dl}$ debido al efecto patológico de la hidrargiria en murinos se muestran en la tabla 6 . Donde se realizaron tratamientos en tres grupos experimental y uno control. Cabe mencionar que cada grupo estuvo constituido por 3 ratones, a los cuales se les aplicó $1 \mathrm{ml}$ de bicloruro de mercurio 1mg para el I Grupo, $2 \mathrm{mg}$ para el II grupo y $3 \mathrm{mg}$ para el III grupo por vía oral, según la metodología correspondiente, luego de los 3 días se hizo la primera lectura de los grupos experimentales, el grupo I tuvo una concentración de creatinina $0.3 \mathrm{mg} / \mathrm{dl}$, el grupo II $0.7 \mathrm{mg} / \mathrm{dl}$ y el grupo III $0.6 \mathrm{mg} / \mathrm{dl}$. La segunda lectura se realizó a los 6 días con el siguiente resultado: grupo I $0.4 \mathrm{mg} / \mathrm{dl}$, el grupo II $0.8 \mathrm{mg} / \mathrm{dl}$ y el III con 0.9 $\mathrm{mg} / \mathrm{dl}$. La tercera lectura se realizó el día 9: grupo I incremento a $0.6 \mathrm{mg} / \mathrm{dl}$, el grupo II a $0.9 \mathrm{mg} / \mathrm{dl}$ y el III grupo $1.0 \mathrm{mg} / \mathrm{dl}$. Es decir que a medida que pasa el tiempo se aumenta los niveles de creatinina, lo que significa que el mercurio se acumula en los órganos dañándolos hasta generar complicaciones, se debe tomar en cuenta el tamaño de los ratones de $15 \mathrm{~cm}$ y el peso va entre 12 a 22 gramos, la edad promedio fue de 50 días.

Esta prueba nos da la información importante sobre el funcionamiento de los riñones, como sabemos la creatinina es un producto químico de desecho producido por 
metabolismo celular, un riñón saludable filtra la creatinina y otros productos de desecho de la sangre y luego salen por la orina. Que sucede cuando los riñones no funcionan bien, entonces se acumula mayo cantidad de creatinina en la sangre, por acción de algunas sustancias dañinas como los metales pesados entre ellos el mercurio. ${ }^{26}$

Es decir que las sustancias toxicas dañan el funcionamiento renal y generan consecuencias como fallas, necrosis y alteraciones de la creatinina.

Escala. J (2017), en Arequipa evaluó el efecto de la toxicidad crónica del metil mercurio sobre los niveles de creatinina y alteración histológica del riñón de Rattus norvegicus variedad Spargue Dawley. Administrando mayor cantidad de mercurio 40 $\mathrm{mg} / \mathrm{kg} /$ día en el tiempo de 30 días. ${ }^{27}$

Algunos autores realizaron trabajos con otras muestras de animales como peces, moluscos y mariscos

Osores. F (2016) utilizó nuevas técnicas como el empleo del microondas o liofilización como procedimientos alternativos de secado de muestra, y el extractante TMAH puede conducir a errores en la determinación de las especies de Hg por pérdidas de las especies volátiles o formación artificial de dimetilmercurio, respectivamente. ${ }^{7}$

Singh et al (2010); Li et al (2015). Algunas especies tales como ostras, mariscos y moluscos acumulan el cadmio proveniente del agua en forma de péptidos ligadores hasta alcanzar valores de concentración entre 100 y $1000 \mu \mathrm{g} / \mathrm{kg}$. En la carne, el pescado y frutas se han reportado valores de concentración entre 1 y $50 \mu \mathrm{g} / \mathrm{kg}$ y en algunos granos entre 10 y $150 \mu \mathrm{g} / \mathrm{kg}^{24}$

Referente a la gráfica sobre los niveles de creatinina $\mathrm{mg} / \mathrm{dl}$ resultado del efecto patológico de la hidrargiria en murinos se plasman en la figura 1. Observando como los grupos I y II solo incrementan ligeramente, mientras que el grupo III acumula mas mercurio para generan valores altos de creatinina.

Generalmente los resultados de los análisis de creatinina en sangre en humanos se miden en miligramos por decilitro o en micromoles por litro. El intervalo normal para la creatinina en sangre puede ser de 0,84 a 1,21 miligramos por decilitro $(74,3$ a 107 micromoles por litro), si bien esto puede variar de un laboratorio a otro, entre hombres y mujeres, y según la edad. Considerando que la cantidad de creatinina en sangre aumenta con la masa muscular, los hombres usualmente tienen niveles de creatinina superiores a las mujeres. ${ }^{26}$

Si el nivel de creatinina sérica es superior al normal, es posible que el médico recomiende confirmar los resultados con otra prueba de orina o análisis de sangre. Si el 
daño renal es una inquietud, es importante controlar cualquier trastorno que pueda contribuir al daño. Es especialmente importante controlar la presión arterial, lo que a menudo requiere tomar medicamentos. No puedes revertir el daño renal permanente, pero con un tratamiento adecuado puedes prevenir daños mayores. ${ }^{26}$

La OEC (2004) realizó estudios de absorción cutánea se han venido realizado in vivo, tanto en animales como los cobayos se rasura la piel y se inyecta intradérmicamente el producto junto con adyuvante de Freund para potenciar el efecto sensibilizante. Una semana después se aplica el producto tópicamente y se mantiene durante 48 horas mediante un vendaje oclusivo. A los 21 días se vuelve a aplicar y se mantiene durante 24 horas; después de retirar el apósito y el producto, se valora la aparición de edema o eritema luego de 48 y 72 horas de su aplicación. El ensayo es largo, ya que se tarda más de 21 días y requiere el uso de numerosos animales, entre controles y tratados, según el procedimiento 427 de la OECD. ${ }^{28}$

Vega. M (2002). Realizó una tesis de caracterización de los bioterios utilizados en la investigación científica. Los compuestos de mercurio inorgánico probablemente son absorbidos desde el tracto gastrointestinal humano hasta un nivel inferior al 10\%, en promedio, pero la variación individual es considerable. ${ }^{22}$

Referente a la sintomatología observada en murinos luego de la administración de las dosis correspondientes se nota en la tabla 7 . Donde el $44.45 \%$ corresponde a la presencia de edemas por la aplicación subcutánea de los ratones y su lenta absorción, en los primeros días presentaron anuria en algunos murinos, el 33.33\% se notaron alteraciones nerviosas, como ataxias, temblores y deficiencias respiratorias. El 22.22\% se observaron daños en la piel, una decoloración interna bajo el efecto del mercurio. Lo que se puede señalar que un alto nivel de que los riñones no funcionan bien. El nivel de creatinina aumenta de manera temporal si estas deshidratado, porque se tiene bajo volumen de sangre. El sistema nervioso central es el órgano crítico para la exposición al vapor de mercurio. La exposición subaguda ha dado origen a reacciones psicóticas caracterizadas por delirio, alucinaciones y tendencias suicidas. La exposición a bajos niveles durante periodos largos se ha asociado a síntomas de eretismo menos pronunciados. Los efectos del mercurio se habían comunicado sólo las dosis más elevadas que las asociadas a la aparición de signos y síntomas del sistema nervioso central.

Vinardell. M (2007), realizó la experimentación toxicológica en animales. ${ }^{3}$ 
Ruiz. I (2016) aplicó una metodología para determinar mercurio en peces a nivel muscular. ${ }^{4}$

Tirado L. (2015). Niveles de metales pesados en muestras biológicas y su importancia en salud. ${ }^{12}$

Cabañero Ana, observó la acumulación e interacción de mercurio y selenio en tejidos animales: desarrollo de nuevas metodologías de análisis. ${ }^{2}$

Li et al (2010). Manifiesta que la neurotoxicidad genera temblores y pérdida de sensibilidad en dedos de ambas extremidades, ataxia, perdida de visión, audición, espasmos y finalmente coma y muerte, estas sustancias mercuriales en animales provocan coagulación de la mucosa gastroentérica, como también lesión en el riñón. ${ }^{29}$

Argumedo et al (2013) determinó la concentración de mercurio total por espectrometría de absorción atómica por vapor de frio, las concentraciones altas de mercurio se observaron en Sus scrofa 8156,9 ng/g pesos seco, seguido de Gallus domesticus 3391,9 ng/g y Cairina moschata 1426, $5 \mathrm{ng} / \mathrm{g}$. el consumo de estos animales en las minas de Santa Cruz representa un riesgo preocupante..$^{30}$

La necropsia realizada en la fisiología sistémica del grupo experimental de murinos afectados por la hidrargiria en la tabla 8 . Se han notificado efectos en el riñón con niveles de exposición del 66.67\%. Los estudios experimentales con animales han demostrado que el mercurio puede inducir patologías autoinmunes en todas las especies ensayadas, pero no en todas las estirpes, lo que indica una predisposición genética. Una de las consecuencias de la etiología inmunológica es que, en ausencia de estudios de la dosis/respuesta en grupos de individuos inmunológicamente sensibles.

Internamente se ha observado una afección pulmonar en un $22,22 \%$ lo que ha generado la dificultad respiratoria en los murinos y los movimientos involuntarios, entre otros. El 11.11\% de murinos presentaron hemorragias internas a nivel gastrointestinal y cardiovascular, debido a los efectos del mercurio que por vía sanguínea llegaron al enfrentarse al tubo gastrointestinal y se asociaron a los ácidos y sustancias alcalinas.

Osores. F. (2016) señala que: de las autopsias realizadas en humanos no expuestos profesionalmente, pero con un número variable de obturaciones con amalgama, se desprende que alrededor de 25 superficies de amalgama en promedio, pueden aumentar la concentración de mercurio en el cerebro en unos $10 \mu \mathrm{g} / \mathrm{kg}$. El aumento en el riñón, basado en un número muy limitado de análisis, es probablemente de 300-400 $\mu \mathrm{g} / \mathrm{kg}$. Sin embargo, la variación individual es considerable. ${ }^{7}$ 
Aymaz. S (2001), manifiesta que el mercurio induce a una nefropatía membranosa a largo plazo debido a pequeñas concentraciones, generando un daño renal. Se ha esclarecido que el síndrome nefrótico posterior a la exposición con mercurio genera una reacción inmune por metales pesados, generando la producción de citocinas. ${ }^{31}$

\section{Conclusiones}

1. Dentro de los metales pesados el mercurio es uno de ellos elementos químicos mas tóxicos directa $\mathrm{e}$ indirectamente, los niveles de creatinina fueron incrementándose de 0.30 a $0.6 \mathrm{mg} / \mathrm{dl}$ en el primer grupo experimental, de 0.7 a 0.9 $\mathrm{mg} / \mathrm{dl}$ y en el tercer grupo de 0.6 a $1.0 \mathrm{mg} / \mathrm{dl}$, generando un conjunto de anomalías en los murinos, por tu tamaño los murinos colapsan rápidamente.

1. Observamos que la intoxicación por mercurio presenta alteración y déficit alterando el funcionamiento del organismo del murino al generar edema $44.45 \%$, alteraciones nerviosas $33.33 \%$ y decoloración de la piel $22.22 \%$

1. En la necropsia realizada se observó que los murinos presentaron daño renal $66.67 \%$, afección pulmonar 22,22\% y hemorragias internas $11,11 \%$

\section{Referencias bibliográfica}

Argumedo, G. Vidal J. Marrugo J. Mercurio en animales domésticos en mina Santa Cruz. Universidad del Caribe. 2013. Colombia.

Arnous, O et al. (2015). Heavy metal riks assessment in wáter a bottom sediments of lake Manzala. Árabe Journal geosciences. Egypt 8 (10). Http//dx.doi.org/10.1007/s12517-014-1763-6

Arriola, P. (2013). Monitoreo químico toxicológico reporte estadístico del laboratorio químico toxicológico. Ins. Nac. Salud, año 19 (11-13). Lima.

Aymaz S, Gros O, Krakamp B, et al. Membranous nephropathy from exposure to mercury in the fluorescent-tuberecycling industry. Neprol Dial Transplant 2001; 16: 2253-2255.

Bakir, F. Daluji S. Murtadha M. Clarson, T. Smith, J. Doherty, R. 1973. Methyl mercury poisoning in Iraq. Science. 181:230

Cabañero, A. (2005). Acumulación e interacción de especies de mercurio y selenio en tejidos animales: desarrollo de nuevas metodologías de análisis. Tesis de doctorado. Universidad Complutense de Madrid. 
CENSOPAS. (2013). Exámenes para la determinación de metales pesados. Bol. Inst. Nac. Salud. Año $20 N^{\circ} 11$. Lima. Punto y Grafía Sac.

Combariza, B. (2009). Contaminación por metales pesados en el embalse de muña y su relación con niveles en sangre de plomo, mercurio y cadmio en el municipio de Cundinamarca. Ppt 115. Tesis de maestría. Universidad Nacional de Colombia.

Escala. J. (2017). Efecto de la toxicidad crónica del metil mercurio sobre los niveles de creatinina y alteración histológica del riñón de Rattus norvegicus variedad Sprague Dawley. Facultad de Ciencias Biológicas. Universidad Nacional de San Agustín de Arequipa. Perú.

Escobar, O. (2010). Bioacumulación de mercurio y selenio en peces pelágicos mayores de la costa occidental de Baja california sur. Instituto Politécnico Nacional. México.

Gaona X. (2004). Mercurio como contaminante global: desarrollo de metodologías para su determinación en suelos contaminados y estrategias para la reducción y su liberación al medio ambiente. Tesis de doctorado. Universidad Autónoma de Barcelona. España.

González, E et al. (2015). El problema de contaminación por mercurio. Rev. Colombiana de nanotecnología y nanociencia.

Huang, $\mathrm{Z}$ et al. (2014). Heavy metals in vegetables and the health risk to population in Zhejiang. Food Control, 36(1), pp. 248-252. Aviable at: doi: http://dx.doi.org/10.1016/j.foodcont.2013.08.036. China.

Li, A. Chan, M. Leung, T. Cheung, R, Lam, T. Mercury intoxication presenting with tics. Archives Health Children, 83(1), 2010. Pp: 174-175

Mayo. Libro de la salud familiar de la Clínica Mayo. 5ta edición. 2018. USA

Nava-Ruiz, C\& Mendez-Armenta, M. (1998). Efectos neurotóxicos de metales pesados (Cd, Pb, Ar, Ta). Archivo Neurociencias, 16(3), pp.140-147.

OECD (2004). Guidelines for the testing of chemicals. $N^{\circ} 437$ : skin absorption in vivo method.

ONU-DAES. 2015. Contaminación por metales pesados. http://. ceida.org/es

Osores, F. (2016). Diagnóstico de salud ambiental humana en la provincia Espinar. Cusco.

Ramírez, A. (2006) Biomarcadores en monitoreo por exposición de metales pesados en metalurgia. Acta. Fac. Med. 17(1). UNMSM. Lima. 
Reyes, Y et, al. (2016). Contaminación por metales pesados: implicaciones en salud, ambiente y seguridad alimentaria.

Ruiz, I. (2016). Metodologías analíticas utilizadas actualmente para la determinación de mercurio en el músculo de pescado. Universidad de Costa rica. Rev. Pensamiento actual. Vol 16-N²6. ISSN: 1409-0112. San José.

Sánchez, C et al. (2010). Perfil sociodemaográfico y epidemiológico de la población expuesta a la contaminación por mercurio, plomo y cadmio. Investig. Enferme., 12(2), pp.93-116.

Sarmiento, M. (1999). Evaluación del impacto de la contaminación del embalse Muña sobre la salud humana. Rev. Salud Pública 1(2), pp.159-171.

Singh, A. (2010). Risk assessment of heavy metal toxicity through contaminated vegetables from waste wáter irrigated área of Varanasi. Tropical ecology, 51(2), pp.3. India.

Tirado, L et, al. (2016). Niveles de metales pesados en muestras biológicas y su importancia en salud. Rev. Nac. Odontol. 2015; 11(21): 83-99. Doi: http://dx.doi.org/10.16925/od.v11i21.895. Colombia.

Vega, M. (2002). (Tesis Universitaria). Caracterización de los bioterios utilizados en la investigación científica. Licenciatura en microbiología y química clínica. Universidad de Costa Rica.

Vinardell, M. (2007). Alternativas a la experimentación animal en toxicología: situación actual. Acta Bioethica. Universidad de Barcelona. España.

WHO. 1978. Declaración de Alma Sata. Suiza. http://who.int/publications/

Wiener. 2000. Protocolo de Creatinina. Wiener laboratories. Rosario Argentina. http://www.wiener-lab.com.ar

York, M et, al. (1998). A criticall review of the assement of eye irritation potential using the rabbit eye. Jornal of applied Toxicology. 52:268. 